\title{
Método para desenvolvimento de práticas de gestão integrada em clusters industriais
}

\author{
Jeniffer de Nadaea, Edwin Vladimir Cardoza Galdamez ${ }^{\text {, }}$ Luiz César Carpinettic, \\ Fernando Bernardi de Souza ${ }^{\mathrm{d}}$, Otávio José de Oliveira ${ }^{\mathrm{e} *}$ \\ ajnadae@gmail.com, UNESP, Brasil \\ bevcgaldamez@uem.br, UEM, Brasil \\ ccarpinet@sc.usp.br, USP, Brasil \\ dfbernardi@feb.unesp.br, UNESP, Brasil \\ e*otaviodeoliveira@uol.com.br, UNESP, Brasil
}

\begin{abstract}
Resumo
0 objetivo deste artigo é propor um método para que as empresas pertencentes a clusters industriais desenvolvam coletivamente, por meio de ações guiadas pela governança, práticas integradas introdutórias de gestão da qualidade, meio ambiente e segurança e saúde do trabalho. Esse método foi desenvolvido com base em uma consistente revisão teórica e em uma pesquisa de campo no cluster metal-mecânico de Sertãozinho-SP, realizada para familiarizar os pesquisadores com o ambiente de estudo e identificar boas práticas e dificuldades de gestão, e, por consequência, aumentar o grau de aplicabilidade da proposta e, por fim, na experiência dos pesquisadores. Esse método está dividido em três macroetapas: (i) preparação/planejamento; (ii) implantação; e (iii) avaliação e manutenção. Cada uma das etapas é composta por ações que devem ser desenvolvidas tanto pelas empresas quanto pela governança do cluster. Acredita-se que o método proposto contribuirá para diminuição das incertezas, custos, tempo do processo e para simplificação dos fluxos de informações e procedimentos dessas empresas.
\end{abstract}

Palavras-chave

Qualidade. Meio ambiente. Segurança e saúde do trabalho. Clusters industriais.

\section{Introdução}

As pequenas e médias empresas (PMEs) têm enfrentado dificuldades que limitam seu desempenho e crescimento, tais como falta de políticas de incentivo, alta competitividade das grandes empresas, dificuldade de obtenção de crédito, atraso tecnológico, obsolescência de equipamentos, baixo investimento em treinamento e fragilidade dos processos de gestão (CARMO; PONTES, 1999; OLAVE; AMATO NETO, 2001; ALBERTIN; TORRES, 2002; BHUIYAN; ALAM, 2005).

Dado esse contexto, a tendência é que elas busquem a formação de alianças para aumentar suas chances de sobrevivência, e isso inclui, por exemplo, sua inserção em clusters industriais (OLAVE; AMATO NETO, 2001; CASAROTTO FILHO; PIRES, 2001).

Esses clusters vêm desempenhando um papel importante para o desenvolvimento regional, o avanço

tecnológico, a promoção de pesquisas científicas e a difusão do conhecimento (SCHIMITZ; NADVl, 1999; MYTELKA; FARINELLI, 2000; BAIR; GEREFFI, 2001). Por esse motivo é importante o desenvolvimento de pesquisas que desenvolvam e/ou adaptem as teorias e os instrumentos de gestão para essa realidade.

Nesse sentido, os sistemas de gestão da qualidade, meio ambiente e segurança e saúde do trabalho são soluções cada vez mais adotadas pelos gestores como forma de identificar e atender as necessidades dos clientes, melhorar o desempenho ambiental e motivar e proteger os recursos humanos, possibilitando o aumento da competitividade empresarial (OLIVEIRA, 2012). Esses sistemas podem ser implantados conjuntamente por meio de um sistema de gestão integrada (SGI). 
O SGl integra todos os componentes e processos de uma empresa, possibilitando que a organização trabalhe em sinergia completa e em uma base de dados única e complementar. Seu objetivo principal é o compartilhamento dos processos, procedimentos e práticas afins (OLIVEIRA, 2012).

Neste trabalho parte-se do pressuposto que a implantação coletiva de ações relacionadas a esses três sistemas (qualidade, meio ambiente e segurança e saúde no trabalho) pode gerar benefícios tanto para as empresas individualmente como para o cluster como um todo, conforme corrobora Nadae (2010).

Logo, a questão de pesquisa que norteou seu desenvolvimento foi: de que forma podem-se desenvolver coletivamente ações integradas introdutórias da qualidade, meio ambiente e segurança e saúde do trabalho em empresas pertencentes a clusters industriais de forma a aumentar sua competitividade?

Pelo exposto, o objetivo deste artigo é propor um método para que as empresas pertencentes a clusters industriais desenvolvam coletivamente, por meio de ações guiadas pela governança, práticas integradas introdutórias de gestão da qualidade, meio ambiente e segurança e saúde do trabalho.

Visando à maior familiarização dos pesquisadores com o objeto de estudo (clusters industriais), de forma a dar um caráter mais aplicado às proposições apresentadas neste artigo, foi realizado um estudo de campo no cluster metal-mecânico de Sertãozinho-SP, no qual se identificaram suas boas práticas e principais dificuldades em relação aos elementos estudados neste trabalho.

De forma a aumentar sua profundidade e exequibilidade, o escopo deste trabalho (delineamento ou condições de contorno) está restrito ao estudo de sistemas de gestão da qualidade, meio ambiente e segurança e saúde no trabalho em empresas inseridas em clusters industriais brasileiros.

Após esta introdução é apresentada uma seção com a síntese da revisão teórica utilizada para realização da pesquisa (2), seguida do método de pesquisa (seção 3). Na sequência têm-se a proposta propriamente dita (seção 4), a conclusão (seção 5) e as referências bibliográficas.

\section{Síntese da revisão teórica}

Nesta seção do artigo será apresentado resumidamente o referencial teórico que subsidiou a formulação do método proposto. Os temas foram selecionados em função de sua ligação e contribuição lógica com o objeto de estudo.

\subsection{Sistemas certificáveis de gestão em clusters industriais}

Os clusters industriais surgem principalmente da necessidade das pequenas e médias empresas (PMEs) formarem alianças e parcerias para se tornarem mais competitivas. Eles vêm sendo importantes para o desenvolvimento regional, avanço tecnológico, difusão do conhecimento e promoção de pesquisas no setor e na região a que pertencem (SCHIMITZ; NADVI, 1999; MYTELKA; FARINELLI, 2000; BAIR; GEREFFl, 2001).

Segundo Porter (1998), cluster é um grupo de empresas e instituições de um setor industrial e mesma área geográfica que se complementam ao longo da cadeia de valor. Pode ser caracterizado pela concentração espacial e setorial de empresas em que o desempenho pode ser explicado pela interdependência entre elas.

0 acirramento competitivo tem levado as empresas a uma busca por métodos de gestão mais eficazes que possibilitem obter qualidade nos processos produtivos e produtos, produzir sem agredir o meio ambiente, promover o desenvolvimento sustentável e melhorar a qualidade de vida dos funcionários.

Tais elementos podem ser conseguidos por meio da implantação de sistemas de gestão da qualidade (SGQ), sistemas de gestão ambiental (SGA) e sistema de gestão da segurança e saúde do trabalho (SGSST). As normas certificáveis ISO 9001, ISO 14001 e OHSAS 18001 surgiram para atender as necessidades do mercado respectivamente em relação aos SGQs, SGAs e SGSST.

Esses sistemas certificáveis de gestão auxiliam no processo de tomada de decisão oferecendo informações mais confiáveis, sucintas e de fácil acesso. Segundo Oliveira (2009), eles têm como principal objetivo dar suporte adequado para a administração criar o futuro desejado para os negócios, estabelecendo objetivos, rumos e estratégias voltadas a produtos e mercados rentáveis, promovendo o desenvolvimento de talentos, assegurando a produtividade e levando à consecução dos resultados empresariais desejados.

Alguns interessantes estudos sobre gestão da qualidade e certificação ISO 9001 em clusters foram desenvolvidos no Brasil. Dentre os quais destacam-se Passos et al. (2002), que estudaram a influência da gestão da qualidade sobre as demandas e os fornecedores do setor metal-mecânico de Camaçari-BA; Silva (2003), que identificou a ausência de sistemas de medidas e a utilização de programas e treinamentos de qualidade no cluster moveleiro de Votuporanga; Souza e Tanabe (2006), que analisaram a motivação, as estratégias, os resultados e as percepções das empresas do cluster de metal-mecânico de Maringá-PR em 
relação à implantação de sistemas ISO 9001; Galdámez (2007), que investigou alguns clusters de calçados e a partir deste estudo desenvolveu um instrumento para medição de desempenho coletivo; e Monteiro (2008), que identificou práticas da qualidade no processo de desenvolvimento de produtos em diferentes empresas de clusters no Paraná.

Somam-se a estes os trabalhos de Gerolamo (2007), que propôs um modelo para gerenciar o desempenho em clusters e redes de cooperação de pequenas e médias empresas; Yamanaka (2008), que apresentou uma proposta para implantação coletiva da 1 SO 9001 para organizações que atuem em clusters; e Yamanaka, Péla e Carpinetti (2008), que discutiram uma abordagem para implantação coletiva da 150 9001 em clusters.

Com relação à gestão ambiental e à $1 S 014001 \mathrm{em}$ clusters, destacam-se os trabalhos de Scarpim et al. (2007), que identificaram as transformações ocorridas no cluster calçadista de Birigui, SP, em função da adoção de estratégias ambientais; Lima et al. (2008), que propuseram um modelo de cooperação interorganizacional para o reaproveitamento de resíduos provindos de indústrias metalúrgicas; e Ferreira (2007), que estudou a gestão ambiental em empresas do polo industrial de Cubatão, SP.

Em relação à gestão da segurança e saúde no trabalho e OHSAS 18001, não se encontraram nas bases de dados pesquisadas, que estão explicitadas no item sobre método de pesquisa, estudos tratando direta e especificamente da sua aplicação em clusters industriais, o que aumenta a relevância e a necessidade da realização de trabalhos como este.

Contudo, identificaram-se alguns trabalhos relacionados ao tema que contribuíram com o método proposto neste artigo. São eles: Carvalho (2002), Goldman (2002), Conforto (2003), Nunes e Franzoni (2004), Souza (2004) e Renner (2007), que consideram a qualidade de vida no trabalho como um fator preponderante para os funcionários tornarem-se mais produtivos, menos alienados e um importante instrumento para diminuir o absenteísmo e patologias ocupacionais.

Os estudos de Pizolotto (2000), Carvalho (2002), Goldman (2002), Nunes e Franzoni (2004), Souza (2004) e Marquetto (2007) tratam das condições físicas pessoais e do ambiente de trabalho, das práticas de prevenção de acidentes, palestras e treinamentos dos funcionários quanto ao uso de EPI (equipamento de proteção individual) e da criação da Cipa (Comissão Interna de Prevenção de Acidentes).

Todos esses estudos foram minuciosamente considerados neste trabalho. Contudo, apesar da sua contribuição científica, ainda se verifica uma interessante lacuna de conhecimento para que se proponha uma abordagem para desenvolvimento de práticas integradas da qualidade, meio ambiente e de segurança e saúde no trabalho em empresas que atuem em clusters.

A seguir são apresentadas as principais contribuições pontuais desse item do referencial teórico para a proposta deste estudo:

- Realizar diagnóstico sobre a existência de elementos da qualidade, meio ambiente e de segurança e saúde do trabalho nas empresas do cluster,

- Planejar ações de desenvolvimento continuado dos elementos básicos dos sistemas certificáveis de gestão (conscientização, capacitação, ferramentas para solução de problemas, padronização, indicadores de desempenho, entre outros);

- Estabelecer políticas, objetivos, metas e matriz de responsabilidade comum aos três sistemas;

- Formalizar e escriturar os processos;

- Desenvolver instrumentos para melhoria contínua;

- Criar sistema de avaliação geral dos processos sob o prisma dos três sistemas;

- Identificar aspectos e impactos ambientais;

- Conscientizar e treinar o pessoal para as questões ambientais e de segurança e saúde do trabalho;

- Desenvolver instrumentos para eliminar, reduzir ou reciclar resíduos;

- Identificar riscos, condições inseguras e atos inseguros;

- Melhorar o ambiente de trabalho e fornecer equipamentos de proteção adequados; e

- Criar um sistema eficaz de prevenção dos acidentes a partir do monitoramento e melhoria de processos, infraestrutura e treinamentos.

\subsection{Integração de sistemas de gestão}

A integração dos sistemas de gestão da qualidade, meio ambiente e segurança e saúde no trabalho pode auxiliar as organizações a reduzir custos de sua implantação, pois implementar sistemas separadamente geralmente representa desperdício de recursos e desgaste dos envolvidos. Outras vantagens são a simplificação do fluxo de informação, redução do custo de treinamentos, diminuição do tempo de adaptação ao novo ambiente de trabalho e otimização dos procedimentos (MOREIRA, 2001; BERNARDO et al., 2009).

Bernardo et al. (2009) sumarizaram (ver Figura 1) e analisaram vários métodos e modelos de integração. São eles: Seghezzi (1997), Wilkinson e Dale (1999), Kirkby (2002), Karapetrovic (2002), Karapetrovic e Jonker (2003), Beckmerhagen et al. (2003), Pojasek 


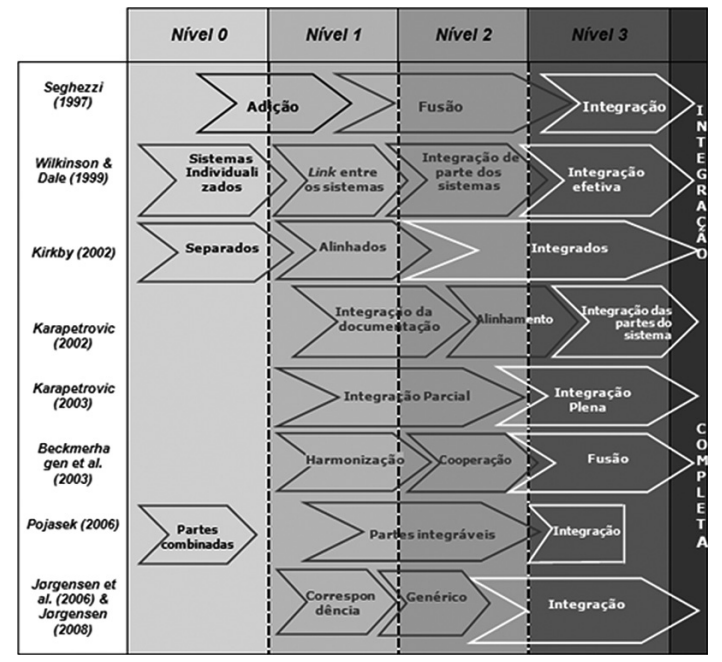

Figura 1. Principais modelos de integração de sistemas. Fonte: adaptado de Bernardo et al. (2009).

(2006), Jørgensen, Remmen e Mellado (2006) e Jørgensen (2008).

Como pode ser observado na Figura 1, a maioria dos modelos sugere três fases contínuas para integração dos sistemas, que de uma forma geral podem ser descritas como alinhamento, cooperação/fusão e integração propriamente dita. Esses trabalhos foram devidamente considerados quando da formulação da proposta apresentada neste texto.

Nesse sentido, as principais contribuições desses trabalhos para a proposta aqui apresentada são:

- Verificar as intersecções, complementaridades e antagonismos entre os sistemas que se pretende integrar;

- Desenvolver a integração de forma evolutiva e contínua, utilizando o ciclo PDCA (Plan, Do, Check e $A c t$;

- Utilizar a conscientização e o treinamento como base para a integração; e

- Fundir documentação, políticas, objetivos, metas, treinamentos e avaliações dos sistemas a serem integrados.

\section{Método de pesquisa}

A proposta apresentada neste artigo é fruto de contribuições da literatura científica somada à investigação que os autores fizeram no cluster metalmecânico de Sertãozinho-SP, que teve como principal objetivo se ambientarem com o tema e conhecerem melhor as características genéricas de aglomerações industriais, ampliando suas percepções e aumentando o grau de aplicabilidade da proposição de um método para desenvolvimento de práticas introdutórias de gestão integrada. Consideraram-se, portanto, as boas práticas lá identificadas, bem como as principais dificuldades em relação à gestão coletiva e individual.

0 referencial teórico foi estruturado visando contemplar os principais elementos constantes no escopo da pesquisa (clusters, qualidade, meio ambiente, segurança e saúde do trabalho e integração de sistemas de gestão). Para sua confecção utilizaram-se principalmente as seguintes bases de dados: Emerald, DirectScience, Pro-Quest, Scielo, Portal de periódicos da Capes, sites de teses e dissertações das principais universidades brasileiras e dos principais congressos da área de Engenharia de Produção e Administração nacionais e internacionais.

A escolha desse cluster foi intencional e motivada pela potencial contribuição que ele poderia dar à pesquisa em função do prévio conhecimento de sua realidade a partir de pesquisas anteriores realizadas por alguns dos autores deste artigo, pela permissão para acesso às empresas e à sede da governança, bem como à documentação pertinente ao estudo (plano de desenvolvimento do cluster, histórico de eventos, planos de treinamento, atas de reuniões, planilhas de indicadores etc.).

Conforme sugerem Yin (2010) e Miguel (2007), foi utilizado mais de um instrumento concomitante para coleta de dados: entrevistas semiestruturadas com integrantes da governança do cluster (diretor do Ceise, Centro Nacional das Indústrias do Setor Sucroalcooleiro e Energético, e gerente do Sebrae, Serviço Brasileiro de Apoio às Micro e Pequenas Empresas - Regional Sertãozinho) e, pelo menos, um gestor de cada empresa estudada (ver roteiro em anexo); visita in loco à sede da governança e de algumas empresas; e análise de documentos. Foram feitas cinco visitas ao cluster, quando também se visitaram as empresas.

0 estudo de campo foi realizado junto a 20 empresas, quando foram realizadas as entrevistas semiestruturadas com vistas a caracterizar sua estrutura e seus principais processos relacionados à qualidade, meio ambiente, segurança e sua integração, e também para identificar e compreender a influência das ações da governança sobre esses elementos.

Este estudo auxiliou no conhecimento do ambiente em que as empresas estão inseridas, suas necessidades e dificuldades e o papel que o agente de governança pode desenvolver na coordenação das atividades e no desenvolvimento do aglomerado.

Os resultados da pesquisa de campo, realizada por meio dos instrumentos de coleta de dados citados, que permitiu entender a dinâmica da interação entre as empresas entre si e das empresas com a governança 
do cluster, somado ao referencial teórico, contribuíram para estruturação do método proposto nesta pesquisa.

\section{Resultados do estudo de caso}

Serão apresentados apenas os elementos principais do estudo no cluster metal-mecânico de Sertãozinho, $\mathrm{SP}$, tais como sua caracterização, principal projeto de melhoria, principais dificuldades e boas práticas na gestão coletiva e individual das empresas.

Existem 261 PMEs do setor metal-mecânico em Sertãozinho que fornecem serviços e produtos às grandes usinas de cana de açúcar instaladas na região. Dessas, apenas 20 participam efetivamente de ações coletivas relacionadas ao cluster metal-mecânico desde 2007. Por isso, apenas elas participaram desta pesquisa.

0 Ceise e o Sebrae exercem a efetiva governança e diretamente coordenam todos os projetos e ações de estruturação e melhoria do aglomerado. Em 2007 esses dois órgãos iniciaram e financiaram o principal projeto interno de melhoria do cluster com o objetivo de capacitar, organizar e estruturar tanto as empresas como os prestadores de serviços.

Ele foi dividido em três etapas principais: (i) produção e tecnologia; (ii) gestão empresarial; e (iii) acesso a mercados e cooperação. Ele ainda está em andamento e tem gerado importantes resultados para 0 cluster, tais como incremento do nível tecnológico dos produtos e processos produtivos, melhor compreensão das necessidades dos clientes, organização coletiva para melhoria da qualidade, formulação, ainda que insipiente, de plano coletivo de marketing, criação de um selo verde para as empresas ambientalmente responsáveis, dentre outros.

Seus procedimentos foram desenvolvidos por meio de capacitação de equipes de trabalho compostas por representantes das empresas, que posteriormente tinham a responsabilidade de divulgar e fomentar a aplicação dos conceitos para todos os funcionários.

Como principais dificuldades, observaram-se falta de política consistente de apoio às empresas, falta de participação efetiva dos demais atores do cluster (instituições financeiras, governo, comunidade científica e fornecedores/compradores), ausência de cooperação efetiva entre os empresários (desconfiança na troca de informações), deficiência na integração das empresas com as universidades e centros de pesquisa, e baixa preocupação com a segurança e saúde do trabalho.

Principais contribuições do estudo de caso para a proposta:

- Macrovisão da gestão do cluster e seu alinhamento com as necessidades internas das empresas;
- Foco no desenvolvimento tecnológico e de gestão;

- Investimento na confiança e cooperação dos empresários;

- Divulgação coletiva da imagem do cluster visando aumento de mercado;

- Preocupação e desenvolvimento de procedimentos básicos da gestão da qualidade e gestão ambiental; e

- Desenvolvimento e implantação evolutiva (em módulos) de projetos de melhoria.

\section{Proposta de método para desenvolvimento de práticas introdutórias de gestão integrada em clusters industriais}

Este trabalho apresenta uma proposta de método para desenvolvimento de práticas integradas introdutórias de gestão da qualidade, meio ambiente e segurança do trabalho em empresas que atuem em clusters industriais elaborado com base no referencial teórico, nas informações obtidas no estudo de campo e na experiência dos pesquisadores.

0 método foi pensado para ser de simples aplicação, direto e adequado à realidade das PMEs. Ele está subdividido em três fases principais: (i) planejamento/preparação; (ii) implantação; e (iii) avaliação e manutenção, esquematicamente apresentadas na Figura 2. Essa estrutura foi baseada nas recomendações da maioria dos estudos analisados por Bernard et al. (2009), já apresentados na síntese do referencial deste texto.

As ações de planejamento, adaptação, implantação e controle das fases devem ser coordenadas pelos agentes de governança. Cada uma dessas fases é composta por etapas, cujo fluxo é apresentado na Figura 3.

0 estabelecimento das etapas do método e o seu sequenciamento (Figura 3), que serão mais bem descritos nos itens seguintes, foram baseados nas fases

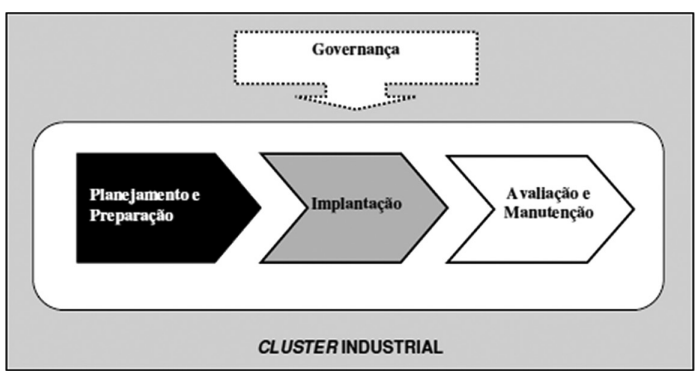

Figura 2. Fases do método para desenvolvimento de práticas integradas de gestão. Fonte: autores. 


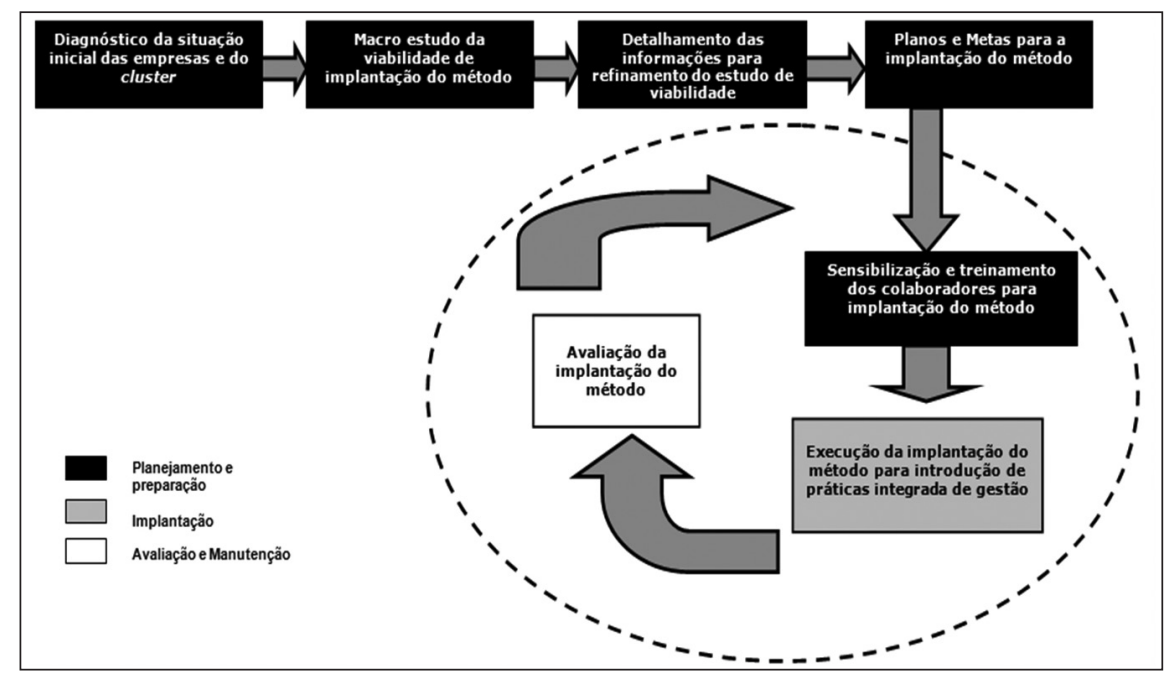

Figura 3. Fluxo de implantação. Fonte: autores.

propostas na Figura 2, na literatura e na pesquisa de campo, principalmente nas entrevistas com o diretor do Ceise e o gerente do Sebrae, que promovem continuamente projetos de melhoria nas PMEs.

\subsection{Etapa de planejamento e preparação}

Nessa etapa a governança deve pensar nos objetivos da implantação do método, seus benefícios, suas possíveis dificuldades, quais e quantos recursos serão despendidos etc. Deve também divulgar todas as fases e informações necessárias para o cluster se envolver como um todo e para sanarem-se eventuais dúvidas.

Deve-se ter grande esforço inicial na sensibilização de todas as empresas e seus funcionários de forma que se reduza a resistência à implantação do método. As empresas podem contratar uma consultoria externa para auxiliar no processo caso julguem necessário.

Deve-se realizar um diagnóstico da situação inicial das empresas e do cluster, cujo objetivo é ter uma visão real das condições existentes para facilitar o desenvolvimento do método proposto. Esse diagnóstico deve verificar quais ações coletivas já foram implantadas e o seu resultado, e se existem recursos físicos, humanos e financeiros adequados.

Deve-se realizar um macroestudo da viabilidade de desenvolvimento do método de forma que a governança consiga prever a ordem de grandeza do quanto será investido durante o período de desenvolvimento, o tempo que levará e a necessidade de manutenção dessas ações.

Os treinamentos devem ser planejados nests fase e devem considerar quais tópicos devem ser abordados, a duração e o número de participantes. Cabe à governança, em comum acordo com as empresas, decidir quais temas são mais relevantes e o tempo de duração de cada palestra, treinamento ou minicurso.

É necessário que se confeccione um plano com metas mensuráveis e factíveis para a implantação do método. Um cronograma de execução deve ser estabelecido em reuniões entre os envolvidos. Cada empresa terá suas metas e o cluster também deve ter as suas de forma que se consiga atingir a plena implantação do método.

Para que tudo ocorra no período planejado, a governança deve se encarregar de controlar o macrocronograma, cobrando e apoiando as empresas para desempenharem suas atividades no prazo estabelecido. Os agentes de governança devem enfatizar sempre os objetivos da implantação e os benefícios que as empresas conseguirão para que todos se mantenham sempre motivados.

As principais ações previstas para essa etapa são apresentadas na Figura 4.

\subsection{Etapa de implantação}

Nessa fase a governança deve auxiliar as empresas no processo de implantação do método, na documentação dos processos, atendimento aos requisitos estabelecidos, esclarecer possíveis dúvidas e verificar se os prazos estão sendo cumpridos por todas elas. Essa etapa é composta por várias ações, descritas na Figura 5, que as empresas e o cluster devem executar tanto isolada quanto conjuntamente.

As empresas devem monitorar suas atividades para garantir que sejam desenvolvidas de acordo com o estabelecido no item anterior (planejamento e 


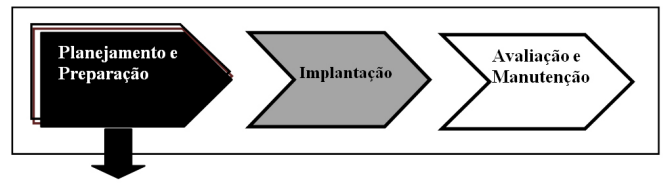

$\checkmark$ Mapear a situação inicial das empresas e do cluster,

$\checkmark$ Verificar a viabilidade da implantação do método;

$\checkmark$ Promover reuniões entre a governança e os empresários;

$\checkmark$ Administrar o impacto da implantação na cultura organizacional (gestão da mudança);

$\checkmark$ Escolher um representante-líder dos empresários;

$\checkmark$ Estimar o valor financeiro macro e os recursos que serão investidos na implantação;

$\checkmark$ Criar estratégias para atingir o objetivo do método;

$\checkmark$ Estabelecer metas para as empresas e para o cluster,

$\checkmark$ Criar mecanismos para minimizar o impacto negativo na cultura organizacional;

$\checkmark$ Oferecer incentivos para os colaboradores durante todo o processo de desenvolvimento do método; e

$\checkmark$ Estabelecer período e temas para a jornada de palestras, treinamentos e minicursos.

Figura 4. Ações da fase do planejamento e preparação. Fonte: autores.

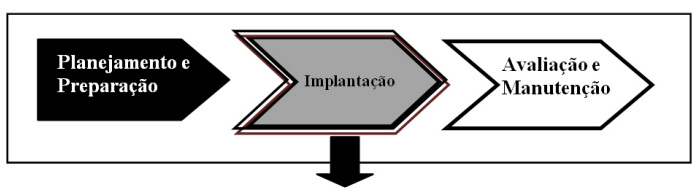

Fornecer infra-estrutura para ações de desenvolvimento das ações integradas

$\checkmark$ Instituir Círculos de Controle da Qualidade ou times de melhoria;

$\checkmark$ Criar meios para disseminar a comunicação interna de todas as etapas de desenvolvimento das ações integradas;

$\checkmark$ Implantar indicadores de desempenho para a introdução das ações integradas;

$\checkmark$ Desenvolver programas de ações corretivas e preventivas para a implantação das açōes integradas;

$\checkmark$ Desenvolver um sistema de gestão que registre todos os processos da implantação das ações integradas:

$\checkmark$ Documentar as instruções e rotinas de trabalho registrando cada etapa da implantação das ações integradas;

$\checkmark$ Criar um macro fluxo dos principais processos de cada empresa, facilitando a identificação de gargalos e facilitando o processo de implantação das ações integradas:

$\checkmark$ Criar um programa de avaliação da satisfação dos clientes internos e externos;

$\checkmark$ Cumprir a legislação ambiental e de segurança no trabalho pertinente facilitando a adequação à implantação das ações integradas;

$\checkmark$ Desenvolver mecanismos de mapeamento e identificação continuada dos requisitos dos clientes internos em relação à segurança e saúde no trabalho;

$\checkmark$ Identificar e mapear os riscos ocupacionais aos trabalhadores, facilitando a implantação das ações de segurança e saúde no trabalho;

$\checkmark$ Identificar os aspectos e impactos ambientais dos processos da empresa, facilitando a implantação das ações ambientais;

$\checkmark$ Criar manual de Boas Práticas de ações integradas de gestão;

$\checkmark$ Desenvolver mecanismos de identificação contínua das necessidades dos clientes internos e externos em relação à qualidade dos produtos ou serviços oferecidos;

$\checkmark$ Oferecer treinamento e palestras sobre programas e ferramentas da qualidade;

$\checkmark$ Implantar programas e ferramentas da qualidade;

$\checkmark$ Monitorar as atividades para que sejam desenvolvidas de acordo com o estabelecido; e

Desenvolver um plano de emergência visando à adoção de procedimentos técnicos e administrativos, estruturados de forma a propiciar resposta rápida e eficiente em situações emergenciais.

Figura 5. Ações da fase de implantação. Fonte: autores. 
preparação). Esse monitoramento pode ser realizado pelo representante-líder de cada organização, que verificará se as etapas e as metas estabelecidas estão sendo atingidas.

Com base no diagnóstico elaborado na primeira etapa, identificar quais empresas têm mais dificuldades humanas, financeiras e estruturais para desenvolver as ações, de forma que se possam fazer correções de rumos a tempo de se cumprir o cronograma estabelecido. Essas ações devem ser antecipadas pela governança e qualquer acontecimento que não esteja dentro do previsto deve ser rapidamente solucionado para que não prejudique o desempenho das demais empresas.

Para facilitar o desempenho dos agentes de governança na coordenação da implantação de cada etapa, é necessário que se desenvolva um sistema documental em que se registrem as instruções e rotinas de trabalho, facilitando a identificação das possiveis duplicações de tarefas, desperdício de tempo e pessoal, tarefas desnecessárias, entre outras. Esse sistema também facilita que as tarefas sejam padronizadas e utilizadas como instruções de trabalho para futuros funcionários da empresa.

Os responsáveis por cada departamento das empresas devem ter o compromisso de registrar ou indicar alguém para elaborar os registros e documentações, de forma contínua, imediatamente após a execução do processo que está sendo controlado. A elaboração de um macrofluxo dos processos de cada empresa também auxilia na identificação de gargalos e facilita o processo de implantação das ações integradas. Esse macrofluxo pode ser desenvolvido pelo time de melhoria com a coordenação do representante-líder.

\subsection{Etapa de avaliação e manutenção}

A governança em conjunto com os representanteslíderes deve verificar se o planejamento está sendo cumprido e identificar quais correções são necessárias. Essa fase contempla as ações apresentadas na Figura 6.

$A$ avaliação da implantação do método deve ser contínua, porém mais intensa no seu final, avaliando todos os registros e documentações, verificando cada etapa de implantação e os resultados obtidos por cada empresa e pelo cluster como um todo.

A avaliação deve ter por objetivo verificar se as empresas implantaram todos os procedimentos previstos, seguiram todos os passos e se estão em processo de melhoria contínua. Nela os agentes de governança devem verificar se os funcionários continuam aplicando o conhecimento e as técnicas aprendidas nos treinamentos, buscando o aperfeiçoamento e aprimoramento dos processos.

Ao final de cada etapa a governança deve elaborar uma avaliação com o intuito de verificar se os objetivos foram atingidos e se a próxima etapa pode ser iniciada. Ao final, se todas as etapas foram cumpridas conforme o especificado, deve-se fazer uma análise do todo, elaborando uma verificação completa sobre o processo de implantação de forma a identificar oportunidades de melhoria contínua do método.

0 método aqui proposto passou por uma avaliação com dois profissionais: um é integrante da governança do cluster onde foi realizado o estudo de campo e o outro é um professor-pesquisador que trabalha com sistemas integrados.

A proposta foi enviada a eles para que a criticassem e sugerissem melhorias. Ambos acharam-na factível e adequada à realidade dos clusters industriais brasileiros e às pequenas empresas, já que nela se privilegiaram a simplicidade dos procedimentos e 0 seu amadurecimento gradativo e contínuo.

O fato de ela já conter sugestões de ações, instrumentos, treinamentos e verificações também foi elogiado. Contudo, ambos solicitaram seu maior detalhamento.

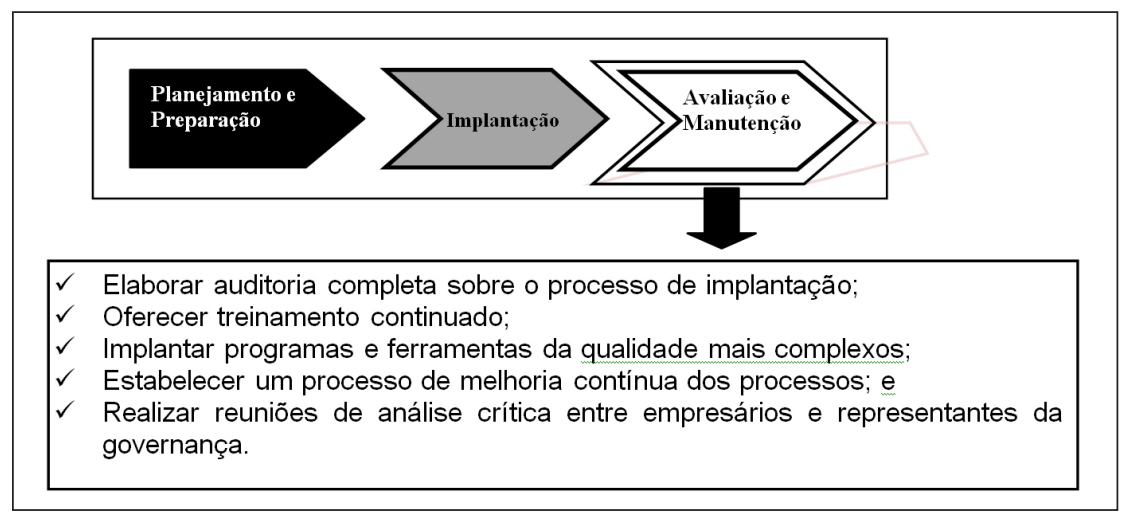

Figura 6. Ações da fase da avaliação e manutenção. Fonte: autores. 
Um deles sugeriu a criação de um manual de boas práticas e que na última etapa, a avaliação do método, fossem adicionadas ações de treinamento continuado aos envolvidos, e outro sugeriu que se verificasse o impacto desse tipo de intervenção na cultura organizacional de forma que a experiência de uma empresa pudesse ajudar as outras.

Essas sugestões e outras mais simples foram devidamente consideradas e incorporadas no método. A proposta apresentada neste trabalho já contempla essas alterações.

\section{Conclusão}

0 principal objetivo deste estudo foi propor um método para desenvolvimento de práticas integradas introdutórias de gestão da qualidade, meio ambiente e segurança e saúde do trabalho a ser implantado coletivamente em empresas pertencentes a clusters industriais. Ele foi alcançado por meio da proposta elaborada com base principalmente na literatura científica apresentada na seção 5 deste artigo, nos resultados do estudo de campo e na experiência dos autores.

Esse método foi estruturado pensando na facilidade de seu desenvolvimento e aplicação, de forma que pudesse efetivamente ser utilizado por empresas com pouca disponibilidade de recursos financeiros e humanos para sua implantação.

Destaca-se que esse método não tem a finalidade principal de certificar as empresas com a 1SO 9001, ISO 14001 e OHSAS 18001, mas implantar práticas introdutórias desses três sistemas, ou seja, ações que se bem desenvolvidas podem servir de base para que futuramente as empresas busquem essas certificações.

Diferentemente dos métodos apresentados na literatura, este estuda a viabilidade de sua implantação e propõe ações para melhoria contínua dos processos rotineiros e das atividades implantadas nas organizações.

Entretanto, tendo em vista que o método ainda não foi testado tal qual foi aqui apresentado, tem-se neste fato uma limitação do estudo que deve ser considerada, mas que também consiste em uma oportunidade para um trabalho futuro.

Por fim, acredita-se que este estudo tenha contribuído com o estado da arte sobre os temas envolvidos tendo em vista que adaptou, customizou e sumarizou as teorias relacionadas direcionando-as para consecução do objetivo proposto. Também tem contribuição prática na medida em que propõe um instrumento para aplicação real em empresas pertencentes a clusters industriais.

\section{Referências}

ALBERTIN, M.; TORRES, M. S. Desenvolvimento de pequenas e médias empresas em clusters. In: ENCONTRO NACIONAL DE ENGENHARIA DE PRODUÇÃO, 2002, Curitiba. Anais... Curitiba: ABEPRO, 2002.

BAIR, J.; GEREFFl, G. Local clusters in global chains: the causes and consequences of export dynamism in Torreon's blue jeans industry. World Development, v. 29, n. 11, p. 1885-1903, 2001. http://dx.doi.org/10.1016/ S0305-750X(01)00075-4

BECKMERHAGEN, 1. et al. Integration of management systems: focus on safety in the nuclear industry. International Journal of Quality and Reliability Management, v. 20, n. 2, p. 209-27, 2003. http://dx.doi. org/10.1108/02656710310456626

BERNARDO, M. et al. How integrated are environmental, quality and other standardized management systems? An empirical study. Journal of cleaner Production, v. 17, n. 8, p. 742-750, 2009. http://dx.doi.org/10.1016/j. jclepro.2008.11.003

BHUIYAN, N.; ALAM, N. A case study of a quality system implementation in a small manufacturing firm. International Journal of Productivity and Performance Management, v. 54, n. 3, p. 172-186, 2005. http:// dx.doi.org/10.1108/17410400510584893

CARMO, V. B.; PONTES, C. C. C. Sistemas de informações gerenciais para programa de qualidade total em pequenas empresas da região de Campinas. Revista Ciência da Informação, v. 28, n.1, p. 49-58, 1999. http://dx.doi. org/10.1590/S0100-19651999000100007

CARVALHO, L. C. R. F. A influência do estilo de liderança na gênese dos DORT em uma fábrica de calçados. 2002. $115 \mathrm{f}$. Dissertação (Mestrado em Engenharia de Produção)Escola de Engenharia, Universidade Federal do Rio Grande do Sul, Porto Alegre, 2002.

CASAROTTO FILHO, N.; PIRES, L. H. Redes de pequenas e médias empresas e desenvolvimento local. São Paulo: Atlas, 2001.

CONFORTO, E. Os determinantes dos resultados das negociações coletivas no setor calçadista do Rio Grande do sul no Período 1996-2001. 2003. 90 f. Dissertação (Mestrado em Ecnonomia)- Faculdade de Ciências Econômicas, Universidade Federal do Rio Grande do Sul, Porto Alegre, 2003.

FERREIRA, L. G. A gestão ambiental do pólo industrial de Cubatão a partir do programa de controle da poluição iniciado em 1983: autores, instrumentos e indicadores. 2007. 289 f. Dissertação (Mestrado em Saúde Pública)- Faculdade de Saúde Pública, Universidade de São Paulo, São Paulo, 2007.

GALDÁMEZ, E. V. C. Proposta de um sistema de medição de desempenho para clusters industriais de pequenas e médias empresas. 2007. 186 f. Tese (Doutorado em Engenharia de Produção)-Escola de Engenharia de São Carlos, Universidade de São Paulo, São Carlos, 2007. PMid:17544433.

GEROLAMO, M. C. Gestão de desempenho em clusters e redes regionais de cooperação de pequenas e médias empresas: estudos de casos brasileiros e alemães e proposta de um modelo de análise. 2007. 227 f. Tese (Doutorado em Engenharia de Produção)- Escola de Engenharia de São Carlos, Universidade de São Paulo, São Carlos, 2007. 
GOLDMAN, C. F. Análise de acidentes de trabalho ocorridos na atividade da indústria metalúrgica e metal-mecânica no estado do Rio Grande do Sul em 1996 e 1997: breve interligação sobre o trabalho soldador. 2002. 151 f. Dissertação (Mestrado em Engenharia de Produção)Escola de Engenharia, Universidade Federal do Rio Grande do Sul, Porto Alegre, 2002.

JØRGENSEN, T. Towards more sustainable management systems: through life cycle management and integration. Journal of Cleaner Production, v. 16, n. 10, p. 1071-1080. 2008. http://dx.doi.org/10.1016/j. jclepro.2007.06.006

JØRGENSEN, T.; REMMEN, A.; MELLADO, M. Integrated management systems - three different levels of integration. Journal of Cleaner Production, v. 14, n. 8, p. 713-22, 2006. http://dx.doi.org/10.1016/j. jclepro.2005.04.005

KARAPETROVIC, S.; JONKER J. Integration of standardized management systems: searching for a recipe and ingredients. Total Quality Management, v. $14, \quad$ n. $4, \quad$ p. 451-9, 2003 . http://dx.doi. org/10.1080/1478336032000047264

KARAPETROVIC, S. Strategies for the integration of management systems and standards. TQM Magazine, v. 14, n. 1, p. 61-7, 2002. http://dx.doi. org/10.1108/09544780210414254

KIRKBY, A. The one-stop shop. Qualityworld, p. 2-4, 2002.

LIMA, S. R. et al. Benefícios da cooperação ambiental interorganizacional: um estudo exploratório em indústrias metalúrgicas gaúchas. Revista Produção Online, v. 8, n. 2, 2008.

MARQUETTO, R. M. F. Integrated management of the environment in the tourism: the route gastronomy of the quarta colônia-RS case. Revista Produção Online, v. 7, n. 3, nov. 2007.

MIGUEL, P. A. C. Estudo de caso na engenharia de produção: estruturação e recomendações para sua condução. Produção, v. 17, n. 1, p. 216-229, 2007. http://dx.doi. org/10.1590/S0103-65132007000100015

MONTEIRO, A. R. G. Gestão da qualidade e do desenvolvimento de produtos nos arranjos produtivos locais de confecções do Paraná. 2008. 242 f. Tese (Doutorado em Engenharia de Produção)- Centro de Ciências Exatas e Tecnologia, Universidade Federal de São Carlos, São Carlos, 2008.

MOREIRA, M. S. 0 desafio da gestão ambiental. Revista Banas Ambiental, São Paulo, n. 10, p. 23-25, 2001.

MYTELKA, L.; FARINELLl, F. Local Clusters, Innovation Systems and Sustained Competitiveness. UNU/INTECH Discussion Paper, out. 2000. Disponivel em: <http:// www.utoronto.ca/isrn/publications/WorkingPapers/ Working01/Mytelka01_Clusters.pdf>. Acesso em: 01 abr. 2011.

NADAE, J. Proposta de método para introdução de práticas integradas de gestão em clusters industriais. 2010. $163 \mathrm{f}$. Dissertação (Mestrado em Engenharia de Produção)Faculdade de Engenharia de Bauru, Bauru, 2010. Disponivel em <http://www2.feb.unesp.br/pos/ bibliotecavirtual/documento.php?COD=a0a080f42e6f13 b3a2df133f073095dd>.

NUNES, E. L. S.; FRANZONI, A. B. Gestão do processo aliada a ergonomia para microempresas. In: ENCONTRO NACIONAL DE ENGENHARIA DE PRODUÇÃO, 2004, Florianópolis. Anais... Florianópolis: ABEPRO, 2004.
OLAVE, M. E. L.; AMATO NETO, J. Redes de cooperação produtiva: uma estratégia de competitividade e sobrevivência para pequenas e médias empresas. Revista Gestão \&t Produção, v. 8, n. 3, p. 289-303, 2001. http:// dx.doi.org/10.1590/S0104-530X2001000300006

OLIVEIRA, 0. J. Gestão da qualidade e produtividade na logística. Curitiba: IESD, 2009.

OLIVEIRA, 0. J. Diretrizes para integração de sistemas certificáveis de gestão da qualidade, meio ambiente e segurança e saúde do trabalho em empresas industriais. 2012. Tese (Livre Docência em Engenharia de Produção)- Faculdade de Engenharia da UNESP, Bauru, 2012.

PASSOS, F. U. et al. Demandas tecnológicas das empresas da cadeia automotiva baiana - um perfil diferenciado. Revista Gestão e Planejamento, v. 3, n. 6, p. 33-41, 2002.

PlZolotT0, M. F. Políticas de benefícios sociais em empresas do setor metal-mecânico do Rio Grande do Sul. 2000. 151 f. Dissertação (Mestrado em Administração)- Escola de Administração,Universidade Federal do Rio Grande do Sul, Porto Alegre, 2000.

POJASEK, R. Is your integrated management system really integrated? Environmental Quality Management, v. 16 , n. 2, p. 89-97, 2006. http://dx.doi.org/10.1002/ tqem. 20124

PORTER, M. Cluster and the new economics of competition. Harvard Business Review, v. 76, n. 6, p. 77-90, 1998. PMid:10187248.

RENNER, J. S. Proposta de um novo sistema de concepção do trabalho no setor calçadista sob a ótica do sistema sócio técnico. 2007. 197 f. Tese (Doutorado em Engenharia de Produção)-Escola de Engenharia, Universidade Federal do Rio Grande do Sul, Porto Alegre, 2007.

SCARPIM, E. P. et al. Gestão ambiental no setor calçadista: estudo desenvolvido nas indústrias de calçados de grande porte de Birigui-SP. In: SIMPÓSIO DE ENGENHARIA DE PRODUÇÃO - SIMPEP, 2007, Bauru. Anais... Bauru: UNESP, 2007.

SCHIMITZ, H.; NADVl, K. Clustering and Industrialization: Introduction. World Development, v. 27, n. 9, p. 15031514, 1999. http://dx.doi.org/10.1016/S0305750X(99)00072-8

SEGHEZZI, H. Business concept redesign. Total Quality Management, v. 8, n. 2-3, p. 36-43, 1997. http://dx.doi. org/10.1080/0954412979677

SILVA, E. M. Alinhamento das estratégias competitivas com as estratégias de produção: estudos de casos no pólo moveleiro de Votuporanga-SP. 2003. 163 f. Dissertação (Mestrado em Engenharia de Produção)- Escola de Engenharia de São Carlos, Universidade de São Paulo, São Carlos, 2003.

SOUZA, J. E. Modelo de avaliação para arranjo produtivo local. 2004. 121 f. Dissertação (Mestrado profissionalizante em Engenharia)-Escola de Engenharia, Universidade Federal do Rio Grande do Sul, Porto Alegre, 2004.

SOUZA, J. P.; TANABE, C. H. Barreiras a implantação da norma ISO 9001:2000 em empresas do setor metal-mecânico da região de Maringá/PR. Caderno de Administração, v. 14, n. 2, p. 46-56, 2006.

WILKINSON, G.; DALE, B. Integration of quality, environment and health and safety management systems: an examination of key issues. Proceedings of the Institution 
of Mechanical Engineers, Part B, Journal of Engineering Manufacture, v. 213, n. 3, p. 275-83, 1999. http:// dx.doi.org/10.1243/0954405991516750

YAMANAKA, L. Proposta para implementação conjunta de um sistema da qualidade 150 9001:2000 em empresas do aglomerado de Sertãozinho. 2008. 285 f. Dissertação (Mestrado em Engenharia de Produção)-Escola de Engenharia de São Carlos, Universidade de São Paulo, São Carlos, 2008.

YAMANAKA, L.; PÉLA, V. R.; CARPINETTI, L. C. R. Gestão de desempenho em aglomerado de pequenas e médias empresas: proposta de um sistema de gestão da qualidade. In: CONGRESSO NACIONAL DE ENGENHARIA MECÂNICA. Anais... Salvador: ABCM, 2008.

YIN, R. K. Estudo de caso: planejamento e métodos. 4. ed. Porto Alegre: Bookman, 2010.

\section{Agradecimentos}

Agradecemos à Capes pelo financiamento da pesquisa e pelas bolsas concedidas.

\title{
Method for introducing collectively integrated management practices in industrial clusters
}

\begin{abstract}
The objective of this paper is to propose a method for introducing collectively integrated practices of quality, environment and occupational safety and health management in companies belonging to industrial clusters. This method was developed based on a literature review. However, field research in a metal-mechanical cluster was also performed to familiarize researchers with the study environment and to increase the degree of proposal applicability. This method is divided into three macro-phases: (i) planning, (ii) implementation and (iii) evaluation and maintenance. Each stage consists of actions that should be developed by both enterprises and the cluster governance.
\end{abstract}

Keywords

Quality. Environment. Occupational safety and health. Industrial Clusters.

Anexo. Roteiro das entrevistas semiestruturadas

1. Quais entidades integram a governança do cluster? As tarefas são divididas? Como?

2. Há um contrato formal para as empresas pertencerem ao cluster?

3. Há metas/compromissos para as empresas que pertencem ao cluster? É

disponibilizado suporte financeiro? Há um planejamento estratégico do cluster? Há um planejamento de marketing? Consultorias externas?

4. Como é avaliado o desempenho de uma empresa e do cluster como um todo?

5. Se uma nova empresa quiser fazer parte do cluster quais os procedimentos

para avaliar e autorizar seu ingresso?

6. Como é feita a avaliação da evolução das empresas no cluster?

7. Há projetos em desenvolvimento ou já desenvolvidos que enfocam práticas

de qualidade, segurança e saúde no trabalho e ambientais? Há integração desses sistemas ou práticas? Como?

8. Quais as ações implantadas que mais impactaram positivamente o cluster e as empresas?

9. Quais os maiores problemas enfrentados no cluster? Quais os pontos positivos do cluster?

10. Existem programas de sensibilização e capacitação?

11. Como se dá a coordenação dos agentes do cluster?

12. Como são definidos os objetivos e metas do cluster?

13. Qual o grau de autonomia de uma empresa que está ligada ao cluster?

14. Já houve algum tipo de exclusão ou afastamento de alguma empresa do cluster? Por quê?

15. De que forma a governança avalia a qualidade dos produtos?

16. Quais as principais dificuldades encontradas na gestão da rede de cooperação?

OBS: As informações foram complementadas pela análise de documentos e observação in loco. 